\title{
Investigating the Interaction Between EEG and HRV Protocol for Study of Three-Circle Post Standing Qigong
}

Jiaxuan Lyu

Beijing University of Chinese Medicine https://orcid.org/0000-0002-5410-6957

Yulong Wei ( $\nabla$ wylbucm@163.com )

Beijing University of Chinese Medicine

Tianyang Tan

Beijing University of Chinese Medicine

Qingchuan Hu

Beijing University of Chinese Medicine

Hangyu Li

Beijing University of Chinese Medicine

Jingjing Dong

Beijing University of Chinese Medicine

Xinzheng Zhang

Beijing University of Chinese Medicine

\section{Study Protocol}

Keywords: Three-Circle Post Standing Qigong, EEG, HRV, complex algorithm, randomized controlled trial

Posted Date: December 31st, 2020

DOl: https://doi.org/10.21203/rs.3.rs-137143/v1

License: (c) (i) This work is licensed under a Creative Commons Attribution 4.0 International License.

Read Full License 


\section{Investigating the interaction between EEG and HRV}

\section{Protocol for study of Three-Circle Post Standing Qigong}

Jiaxuan Lyu ${ }^{1}$, Yulong Wei ${ }^{1 \#}$, Tianyang Tan ${ }^{1}$, Qingchuan $\mathrm{Hu}^{1}$, Hangyu $\mathrm{Li}^{1}$, Jingjing Dong ${ }^{1}$, Xinzheng Zhang ${ }^{1}$.

\section{Affiliations}

1.School of Acupuncture-Moxibustion and Tuina, Beijing University of Chinese Medicine, Beijing.

\section{Abstract \\ Background}

Qigong has a long-term application as an initiative therapy for prevent and cure diseases, as a mindful exercise, it's unique function of integrate mind, breath and body gets a lot of attention, researches show that qigong practice could adjust brain and heart function. Currently we have limitations on study qigong's coordinate mechanism between mind and body, existing studies lack of evidence on electrophysiology research.

Our object here is to propose a method to evaluate the effects of qigong on mind body adjustment, and to analyse the coordinate function of Electroencephalogram(EEG) and Heart rate variability(HRV) by complex signal process.

\section{Methods}

A two-arm randomized clinical trial with eighty-four qigong naïve subjects without cerebral or cardiovascular diseases or other severe syndromes will be allocate to either intervention $\operatorname{group}(n=42)$ or wait in list control group $(n=42)$. Participants in the intervention group will conduct Three-circle post standing qigong exercise 5 times per week for 8 weeks, while wait in list group will maintain their current lifestyle during 8 weeks period. The outcome will be complexity-based measures of heart rate and EEG signals assessed at baseline and 8 weeks. Multiscale entropy analysis will be used as measures of complexity.

\section{Conclusion}

This study will be investigate the effects of qigong exercise by EEG and HRV analyse before and after Three-circle post standing practice, and to measure their synergies by complex signal process method. Results of this study may lead to novel biomarkers that help us monitor and understand the physiological processes of brain and heart function and explore the potential benefits of Qigong and will provide a research evidence for qigong's mind and body synergy function and to direct future clinical treatment.

\section{Ethics and dissemination}


The protocol has been approved by the institutional review boards of Beijing University of Chinese Medicine, where the study will take place(approval number: 2018BZHYLL0109).

\section{Trail registration:}

This study has been registered with the"Chinese Clinical Trail Registry", Republic of China, which is a registry in the WHO Registry Network. Register number: ChiCTR-Bon-17010840.

Keywords: Three-Circle Post Standing Qigong, EEG, HRV, complex algorithm, randomized controlled trial

\section{Introduction}

As we know that our body is a complex system with connects between each two systems, two tissues and two cells. Their dynamic functions consists of our biological system. Malfunction of any part would be a threat to our health. Clinically, we found that mental and physical symptoms always appears together and affect each other, it would have impact on the diagnose and prognoses. However, it's not clear that how they connected and how's their function affect each other. It's hard to observe this connection on a healthy system, but as a mindful exercise, qigong could provide a unique vision for it could make adjustment on both mind and body simultaneously.

Chinese Medical Qigong(CMQ) is a component intervention with complex effects, researches suggest that it have affections on physical and mental dynamic. The regulation on mind and body could act as a complementary prescription for chronic disease rehabilitation, such as cancer recovery ${ }^{1-5}$, post stroke recovery ${ }^{6-10}$ and postoperative recovery ${ }^{11-12}$, to release pain and to restore body function. Another common application is to take CMQ as an alternative therapy. Other than supportive function of clinical conditions, as a preventative and rehabilitative intervention, CMQ can also be used to improving and maintaining health, for example to control blood pressure ${ }^{13-23}$ and glycemia ${ }^{24-27}$, could help build a healthy lifestyle.

There are various types of qigong forms. Since standing position has advantage to stretch our muscles, it could help with flows of our qi and blood. Here we choose the Three-circle Post Standing qigong(TCPSQ), which is a representative static exercise can be traced back to martial arts, is sample and easy to operate for qigong naïve. TCPSQ have character of bilateral symmetrical physical body posture, the balance between left and right side of body, and between front and back side of body is significant. Combined with breath regulation connect inner and outer body, the mind and body can be intentionally linked during practicing, so the integrate of 3 adjustments can be achieved.

Electroencephalogram(EEG) is a nonlinear complex physiology signal with multiple temporal scales, among modern neuroimaging modalities, EEG has drawn extensive attentions to investigate neuronal brain functions and dynamics for it's features of convinent, noninvasive and inexpensive ${ }^{28}$. Since researches proved that EEG could provide functional brain network information, EEG analysis based on complex network of graph theory has been widely used ${ }^{29,30}$. With an intentional qigong prictice, EEG signal could show the changes on one's mind function and brain dynamic. 
Heart rate variability (HRV) is the variation in the time intervals of adjacent heartbeat, can be used to quantify autonomic activities ${ }^{31-32}$. Heart rate rhythms exhibit features of complex systems refecting dynamic, non-stationary, nonlinear properties. HRV can be associated with exercise capacity, as a non-invasive technique, spectral analysis of HRV has been used widely for examining outflows of the heart, which represent the function of sympathetic and parasympathetic nervous system, can also be associated with the presence and the prognosis of cardiac disorders. Therefore, we could investigated and predict adults' exercise capacity with the cardiac function by HRV. Since autonomic nervous system can't be deliberately controlled, it can be adjusted by the breath control. During qigong practice, the mind, body and breath can be regulated at the same time, it's a good chance to learn how intentionally breath regulation affects autonomic nervous system and get to know the merchanism of their coordination.

Both EEG and HRV are nonlinear and non-stationary signals. Traditional linear analyse methods may cause message loss when processing those comlplex signals. In this study, we are going to re-investigate the features of EEG and electrocardiogram(ECG) signals, we will introduce Multiscale Entropy (MSE), one widely used metric of complexity measure for physiologic signals $^{33-34}$, proposed as a measure of the complexity of a physiologic time series ${ }^{35}$, to estimate the nonlinear complexity of across EEG and the dynamic properties of HRV based on two innovative analysis algorithms ${ }^{36-39}$, which could discern features such as spectral and temporal features extracted from the EEG, ECG signals ${ }^{40-43}$.

\section{Methods/Designs}

\section{Study design}

This study is a two-arm, randomized wait in list controlled trial to compare intervention group with wait in list control group for pre- post and parallel comparison between groups. The following outcomes are measured: EEG and ECG. The study design is depicted in Figure 1. 

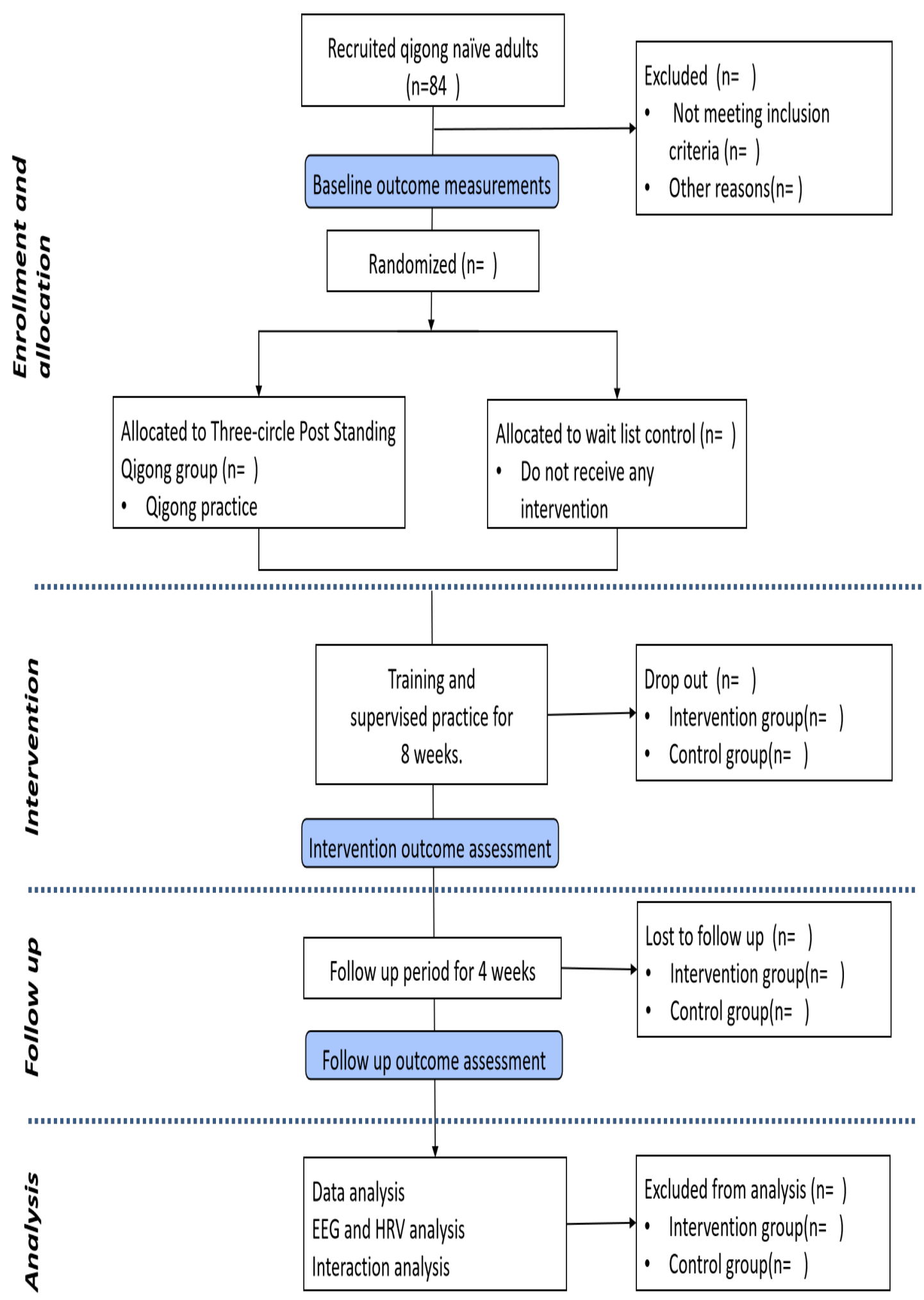

Figure 1 Flowchart of the study design 


\section{Ethics}

The protocol has been approved by the institutional review boards of Beijing University of Chinese Medicine(BUCM), where the study will take place. This study has been registered with the

"Chinese Clinical Trail Registry", Republic of China, which is a registry in the WHO Registry Network. Register number: ChiCTR-Bon-17010840. Registered 12 October 2018 , http://www.chictr.org.cn/edit.aspx?pid=18439\&htm=4.

This study will be monitored by an independent data and safety monitoring board. Advertisements for voluntary subjects will be publish online. All of the participants will provide written informed consent.

\section{Participants}

\section{Inclusion criteria}

The inclusion criteria are as follows:

(1) Volunteers age between 18 and 25 years ${ }^{44}$, right-handed;

(2) Fluency in mandarin and signed Informed consent;

(3) Finish all trainingand test process.

\section{Exclusion criteria}

The exclusion criteria are as follows:

(1) Qigong master or with experience of qigong practice;

(4) Severe mental illness;

(5) History or Family History of mental illness;

(6) Nervous system, respiratory system, cardiovascular and cerebrovascular diseases or taking therapeutic drugs;

(7) Malignant tumors or other serious chronic diseases, infectious disease, or cardiovascular, liver, kidney, gastrointestinal, blood system diseases;

(8) Scalp trauma or Cerebral insufficiency;

(9) Epilepsy or history of epilepsy;

(10) With metal or cardiac pacemaker implants;

(11) Musculoskeletal system diseases or other sports contraindications;

(12) Chronic cardiovascular or cerebrovascular diseases such as hypertension and diabetes;

(13) Participated or were participating in a clinical trial that influenced the evaluation of the study results within the 8 weeks.

\section{Withdrawal criteria}


Participants will be withdrawn from the trial if they present any of the following conditions:

(1) Poor compliance or noncompliance (assessed by mean compliance $<85 \%$ at the last estimation);

(2) Occurrence of a serious adverse events, such as unable to progress because of sudden disease;

(3) Subjects required to exit at any time;

(4) Members in the control group have regularly engaged in TCPSQ or other kind of mindful exercises;

(5) Subjects with severe bioelectrical signals loss or damaged, their data will be withdraw from the group when processing.

\section{Participant recruitment}

We will recruit 84 participants; all participants will provide written consent before enrolled in the study.

The clinical research coordinator (CRC) will initially screen by telephone each potential participant against the inclusion and exclusion criteria. Willing potential participants will be given an appointment at the center for the qigong trial in BUCM. A screening log will be maintained to record the inclusion/exclusion criteria for all those participants. The CRC will briefly explain the purpose of the study and ask if they have an interest in participating. The CRC will also record basic demographic and medical information.

\section{Sample size}

There are no previous clinical trials to evaluate the effect of CMQ on interaction between mind and body. Therefore, this research is designed to follow the EEG and HRV study of TCPSQ to calculate the appropriate sample size for future randomized clinical trials. The TCPSQ treatment group's mean power of EEG alpha band is 18.10 , with the standard deviation is 4.689; while control group with mean EEG power of 17.91, and standard deviation is 4.534. The mean frequencies low frequency in HRV(LF) are 55.57(TCPSQ group) and 54.53(control group), and the standard deviations are 23.63(TCPSQ group) and 21.16(control group). According to aforementioned results, we calculate the sample size by PASS 11.0 (NCSS, LLC. Kaysville, Utah, USA.), set alpha as 0.05 , and the test effectiveness is 0.90 . As the result we will include 35 participants in each group, consider about the $20 \%$ of withdraw rate, we will include 42 subjects for each group, which contains total sample size of 84 subjects for evaluating the effect of qigong's mind body interaction ${ }^{45-48}$.

\section{Randomization and allocation concealment}

The subjects admitted to the study will be randomized to either the intervention group or the wait in list group. Participants will be randomized before the first treatment by an online centralized randomization service. Randomization will be stratified by age and sex.

Allocation concealment will be ensured, as the service will not release the randomization result until the patients are recruited into the trial after all baseline measurements are completed. 


\section{Informed consent}

Prior to the study, general process of the study and the responsibilities of participants and researchers will be explained to all potential subjects. We will confirm that they are volunteered to join the trial and told them they are free to leave if they want to. Written informed consent will be obtained from each participant before any related interventions start. A research assistant will be responsible to obtaining informed consent from all participants.

\section{Collection of demographic information}

Each patient's medical history, including current medication, surgical history, and the presence of other diseases will be recorded at baseline. To investigate other risk factors for cardiovascular diseases or cerebrovascular diseases, lifestyle factors including diet, exercise, smoking, and drinking alcohol will also be documented. Height, weight, age and other demographic information will also be measured.

\section{Blinding}

It will be difficult to blind the participants and Qigong coaches because the wait in list control group will not receive any intervention on section one. So we make strict rule for each investigator to defined their obligation, the recruitment, allocation, Qigong treatment, data collection and analyses will be assigned to different researchers. The allocation sequence and blind code will be preserved by an independent project manager who is irrelevant to all other trail segments, and will be release it until the statistical analysis is completed; the project manager and Qigong coaches can not take part in the assessment of outcome; the outcome assessors, laboratory technicians, data managers, and the statistic analyzer will not allowed to involve in the participants' screening and allocating. Outcome assessors will be blind to group allocation and will not be involved in providing intervention.

\section{Intervention}

\section{Three-circle Post Standing Qigong}

Although there have been several CMQ trials, the type of CMQ used in those trials was diverse. We have developed a TCPSQ protocol for the research of psychosomatic synergy based on the training scheme originated from the TCPSQ recorded in Traditional Chinese Medicine Qigong ${ }^{49}$ (" $13^{\text {th }}$ Five-Year" planning teaching materials of China National Higher Education of Chinese Medicine, Beijing: China Press of Traditional Chinese Medicine. Editor: Tianjin Liu, Wenchun Zhang) and Chinese Medicine Qigong Training Guidance ${ }^{50}$ (Beijing: China Press of Traditional Chinese Medicine. Editor: Yulong Wei). Two qualified coaches with the experience of CMQ education at least 10 years will be participant the TCPSQ intervention period.

Three Circle Standing Qigong will be taught by an instructor with expertise in qigong. The participants will visit the clinic and take at least 5 qigong classes per week for 8 weeks. Each qigong class lasts 50 minutes. The purpose of qigong exercise is to familiar with Three Circle Qigong pracitce, to initiative practice by themselves to integrate mind, body and breath regulation into one.

The compliance of the subjects will be assessed in terms of the number of qigong training and 
practice attended and the feedback from each subject by questionnaires. Every possible effort is made to keep subjects actively engaged in the protocol following attendance and practice guidelines. No medications that affect cardiovascular or cerebrovascular system will be permitted during the study.

\section{Intervention regimen}

TCPSQ training will be consisted by a 3 consecutive half-days initial workshop conducted by 2 qualified coaches, a 8 weeks period of TCPSQ practice under coaches supervised and a 4-week follow-up.

During the initial workshop, participants will receive a training process, which consists of instructions about the source and characteristic of TCPSQ, get to know it's essence and benefits; and the relevant traditional Chinese philosophy and Chinese medicine knowledge relate to TCPSQ, to help them understand and perform TCPSQ. The instruction of TCPSQ will be both verbal and visual. Participants will also be introduced of study procedure and precautions. Participants will also be required to learn key movements known as "regulating body, breathe, mind" and ancillary exercises based on multiple repetitions of TCPSQ until they acquired. This process will be confirmed by the professional coaches. The coaches will be required to supervised each individual to ensure that the movements are being correctly practiced.

Once training process being completed, participants will be asked to practice TCPSQ under supervising. Subjects of TCPSQ intervention group will undergo the 8 weeks period of regular practicing together with a frequency of 5 days per week. Practice will be performed for 50 minutes per day, each session will include a 10 minutes warm-up, a 30 minutes TCPSQ practice, and a cooling down portion for 10 minutes. (Table 1). The instructors will supervise each individual to ensure that the movements are being correctly practiced.

All participants will be required to fill self-made TCPSQ practice self-evaluation scale (including practice difficulty, the feelings of relaxing, pain and fatigue, sweat, saliva secretion, warmth of body, breath control, meditation, physical and mental reactions during and after the training) and record their daily practice reports on the intervention period.

To eliminate the bias of participants extra activities, participants in both groups will be asked to keep a diary, including the type and intensity of physical activity or exercise, as well as daily sedentary time and sleep duration throughout the study.

Table 1 Composition of Three Circle Standing Qigong

\begin{tabular}{cc}
\hline stage & motions \\
\hline Warm-up & Normal state \\
1st & Baseline, steady standing with eyes closed \\
2nd & Separate two feet \\
3rd & Toes slightly point inside, there is a circle of arches between two feet
\end{tabular}


Two arms hold a circle

Hold a circle between two hands

Colse your eyes and take deep, slow and even abdomen breath

Take 3 deep breath and open your eyes

Go back to normal state

\section{Wait in list control group}

The wait in list control group will receive no instruction in first section when intervention group practice TCPSQ, and don't know any details about qigong practice. They will be asked not to participate in any kind of mindful exercises, such as yoga or other forms of Qigong. And to maintain their routine lifestyle, including diet, exercise, work and other activities.

On the completion of the study they will be taken the same 8 weeks of TCPSQ practice after the 12 weeks as the study design.

\section{Follow-up period}

All participants will return to their original lifestyles during the 4-week unsupervised follow-up period. Participants will be required to record their daily physical activities and sport information. Records will be collected by researchers for following up everyday by email or mail.

The physical and psychological self-reported scales will be re-evaluated when follow-up period ended. The follow-up assessment is designed to evaluate the long-term effect of TCPSQ on physical and psychological health, and to act as part of baseline measurement records for wait in list group subjects before their training period start.

\section{Quality control}

(1) Quality control for Researchers:

We will train our researchers before the study begin. All researchers will be asked to memorize the quality and compliance monitoring processes, make sure they are familiar with all study design, and follow the instructions; we will assign the recruitment, allocation, coaches, data collection and analyses work to specific person, then defined their obligations, and strictly prohibited any disclosure between researchers. All the sealed information will be responsible to preserve by the representative researchers until the trial is completed.

We will make assessments on coaches teaching skills before training process, to eliminate the differences of teaching method between coaches, so all subjects could receive same classes from two coaches, the individual-caused difference can be minimized. All researchers will also be trained to unified their intervention or test skill at every stage of the study when they facing participants or communicate with them, to avoid the personal interference bias.

(2)Quality control for participants: 
During the training and supervised practice phase, the researches will check in all participant and summarize it after each training, they will contact the subjects who didn't attend the training, get to know the reasons why they didn't show up, and urging them to finish their training; After each training, the researchers will ask the subjects about their feelings of practice, to scale the quality by their feedback, record their problems on the report forms, and deal with the problems they possible have.

When collect the bioelectronic signals, to avoid the possible interrupt or forgotten of practice for the participants who get later test because of the tests order, the participants will be asked to continue their practice until the end of the individual test.

All participants are required not to share their practice details to the members of wait in list control group in the first period of study, and members of the wait in list group should not practice any form of mindful exercises during the first 12 weeks to act as the parallel non-intervention control. The wait in list control group are promised to get TCPSQ training and supervised practice same with the intervention group after 12 weeks.

\section{Outcome measures}

The primary outcome will be measure central nervous system EEG and cardiovascular ECG before and after qigong exercises. EEG and ECG will be taken at every visit(Table 2). The room temperature and humidity are being controlled, and testing protocol is designed to minimize fatigue and patient burden and maximize the validity of the data collected.

The nervous system function will be collect by a 37-electrode EEG cap with international 10-20 system with linked mastoid reference(NeuroScan Co. USA.), $1000 \mathrm{~Hz}$ sampling rate, high pass filter of $0.05 \mathrm{~Hz}$, low pass filter of $70 \mathrm{~Hz}$, notch filter of $50 \mathrm{~Hz}$, and impedance below $3 \mathrm{k} \Omega$. We recorded 37 electrodes. The signals from these 37 electrodes were referenced to linked mastoid lobe electrodes. All recordings are artifact-free EEG segments of 60 seconds duration. At the stage of data preprocessing, the Independent Component Analysis (ICA) was used to eliminate the artifacts (eyes, muscle, and cardiac overlapping of the cardiac pulsation). Steady-state EEG dynamics are assessed during relaxing standing, and will be collect for 5 minutes. Additionally, a trained research assistant will measure the EEG signal at the same time with the subject in the Three-circle post standing position.

The heart rate(HR) will be collected by MP-150(BIOPAC Co. USA.), $1000 \mathrm{~Hz}$ sampling rate, and allowed a 5 minutes warm-up before test. To collect the sufficient number of heartbeat cycles for complexity measures, HR dynamics are also assessed for 5 minutes simultaneously with EEG at both steady-state and Three-circle post standing state. Results in HR, with change in HR response reflective of cardiovascular regulation will be collect.

Subjects will be instructed to wear loose and comfortable clothing and shoes. The investigators will refrain from wearing laboratory coats during all sessions, as the use of such apparel has been associated with increased heart rate in some patients (white-coat hypertension).

Table 2 Summary of outcome measures and associated variables

\begin{tabular}{|l|l|l|c|}
\hline & Physiological & Testing methods & Outcome \\
\hline
\end{tabular}




\begin{tabular}{|c|c|c|c|}
\hline & measurements & & variable \\
\hline \multirow{2}{*}{$\begin{array}{l}\text { Temporal dynamics } \\
\text { during steady-state } \\
\text { conditions }\end{array}$} & Heart rate & $\begin{array}{l}\text { Beat-to-beat variation } \\
\text { measured using ECG for a } 10 \\
\text { minute during standing relaxing } \\
\text { state }\end{array}$ & HR complexity \\
\hline & EEG & $\begin{array}{l}\text { Using EEG for a } 10 \text { minute } \\
\text { during standing relaxing state, } \\
\text { collect simultaneously with } \\
\text { ECG }\end{array}$ & $\begin{array}{c}\text { EEG } \\
\text { complexity }\end{array}$ \\
\hline \multirow{2}{*}{$\begin{array}{l}\text { Qigong dynamics during } \\
\text { Three-circle post } \\
\text { standing-state conditions }\end{array}$} & Heart rate & $\begin{array}{l}\text { Beat-to-beat variation } \\
\text { measured using ECG for a } 10 \\
\text { minute during standing relaxing } \\
\text { state }\end{array}$ & HR complexity \\
\hline & EEG & $\begin{array}{l}\text { Using EEG for a } 10 \text { minute } \\
\text { during standing relaxing state, } \\
\text { collect simultaneously with } \\
\text { ECG }\end{array}$ & $\begin{array}{c}\text { EEG } \\
\text { complexity }\end{array}$ \\
\hline Protocol adherence & Qigong training & $\begin{array}{l}\text { Teachers report class } \\
\text { attendance and records. } \\
\text { Self-reported practice feelings } \\
\text { and problems. }\end{array}$ & $\begin{array}{c}\text { Hours of } \\
\text { practice/week }\end{array}$ \\
\hline
\end{tabular}

Table 3 Trial measures processes chart. 


\begin{tabular}{|c|c|c|c|c|c|c|}
\hline items & $\begin{array}{l}\text { Baseline } \\
\text { measurement } \\
\text { (week -2 - 0) }\end{array}$ & $\begin{array}{l}\text { Intervention period } \\
\text { (week } 1-8 \text { ) }\end{array}$ & $\begin{array}{l}\text { Intervention } \\
\text { outcome } \\
\text { assessment } \\
\text { (week 9) }\end{array}$ & $\begin{array}{l}\text { Follow up } \\
\text { period } \\
\text { (week } 9-12 \text { ) }\end{array}$ & $\begin{array}{l}\text { Follow up outcome } \\
\text { assessment } \\
\text { (week 12) }\end{array}$ & analysis \\
\hline inclusion criteria & * & & & & & * \\
\hline exclusion criteria & * & & & & & * \\
\hline informed consent & * & & & & & \\
\hline demographic information & * & & & & & * \\
\hline $\begin{array}{l}\text { randomnization and } \\
\text { allocation }\end{array}$ & * & & & & & \\
\hline $\begin{array}{l}\text { bioelectrical activity of } \\
\text { cortical neurons }\end{array}$ & * & * & * & & & * \\
\hline $\begin{array}{l}\text { electrical acticity of the } \\
\text { heart }\end{array}$ & * & * & * & & & * \\
\hline $\begin{array}{l}\text { self evaluation of qigong } \\
\text { training }\end{array}$ & & * & * & & & * \\
\hline self report & & * & * & * & * & * \\
\hline adverse events & & * & * & * & * & * \\
\hline
\end{tabular}

\section{Data analysis}

Collected data will be recorded on standardized forms. The investigators will be supervised and verified when analyses, in order to avoid errors and minimize biases in the process.

Descriptive statistics will be calculated for dependent and independent variables. This analysis will include summary statistics of demographic information and outcome measures. Demographic and clinical characteristics of subjects in the intervention group and the wait in list group will be compared upon admission using a 2-sample t-test (continuous data). To control for baseline differences between two groups, variables that are significantly different at baseline will serve as covariates in the analyses. Data will be analyzed on an intention-to treat basis. IBM SPSS Statistics, version 25.0 (SPSS Inc., Chicago, Illinois, United States) was used for all of the statistical analyses.

The EEG and ECG signals were exported in European Data Format and were processed using MATLAB R2018b (Mathworks Inc., Natick, MA) software was used for statistical evaluations. The MSE algorithm comprises two steps: (1) a coarse-graining procedure that allows us to look at representations of the system' $s$ dynamics at different time scales, and (2) the quantification of the degree of irregularity of each coarse-grained time series, which can be accomplished using sample entropy (SampEn). The complexity index is the summation of the sample entropy values for each coarse-grained time series for a pre-selected range of time scales. A higher MSE score implies greater complexity.

After MSE analysis will applied to each segment in the statistical analysis. For each segment, the difference in the complexity between the two groups (intervention and wait in list) was assessed using one-way analysis of variance (ANOVA) as both normality and homogeneity of variance were not violated. Our analysis will be based on a longitudinal regression analysis in which we do not have an estimate of the within-participant correlations. Nonparametric methods will be used when assumptions of normality are violated. The alpha level will be set at $<0.05$. 
Adverse events will be reported to the data and safety monitoring board. Adverse events will be recorded when participants present with complaints at any time and will be based on the safety policy and adverse event forms.

Serious adverse events will be recorded as required by the International Conference on Harmonization Guide line E2A. We will submit the results of the trial for publication in an appropriate journal irrespective of outcome. We will report the trial in accordance with the CONSORT statements.

\section{Discussion}

Our goals is to compare the change in the timeline in the synergy of EEG and HRV before and after TCPSQ practice between two groups. Here we hypothesize that complexity systems functions are associated with each other. We will compare the connections between complexity measures and function capacity at baseline and 8 weeks later, and to investigate whether changes in EEG are associated with changes in HRV. Since this is a pilot study, we won't make adjustment for multiple testing, and we will include all available data ${ }^{51}$. Our primary analysis will employ nonlinear metric of complexity measure that examine change over time for each outcome measure and for each of the systems. So the primary end point for this study is the change in EEG, HRV and their synergy effects from baseline to 8 weeks post therapy. The primary comparison is between participants randomly assigned to TCPSQ exercises versus wait in list control. Subgroup analyses for age, gender and will be conducted by including each classification. We will use mixed effects models to examine the effects of Qigong practice over time on physical and mental function, and change in heart rate and brain dynamics.

As one of representative mindful exercises, $\mathrm{CMQ}$ is a famous mind-body aerobic practice which originated from Traditional Chinese Medicine ${ }^{44}$, CMQ has been found to have effects on various conditions, such as to prevent and cure psychological and physical disease. CMQ characteristic with initiative exercises to regulate body, breath, mind and to integrate them into one. It's been widely used in clinical for its relaxing and healing effect. Many qigong researchers consider that the qigong effects on the human body are systemic, not only works on body, but also on mind. However what's the mechanism among these two systems and how they related to each other are still not clear. As a initiative exercise, Qigong practice was performed by practitioner themselves, which means qigong is a consciousness-led practice. That's why when practitioner reach the state of oneness with 3 adjustments, the function of body and brain could get regulation simultaneously. A number of questions we facing is that limit of our ability to evaluate complexity-based physiological metrics as biomarkers for monitoring complex system, as well as their value for quantifying the impact of mindful exercises such as Qigong on mind body regulation and their interaction. By MSE analyse for complexity and systematic physiological signals, this study will characterize qigong's impact on moment-to-moment variations in dynamic system, as well as the relationship of physical and mental function.

Physiologic complexity is typically estimated using a number of techniques derived from the fields of nonlinear dynamics and statistical physics that quantify the moment-to-moment quality, scaling, and/or correlation properties of dynamic signals ${ }^{52}$. Compared to other entropy-based methods, MSE is uniquely representative for encode dynamical information in the physiologic signals over multiple scales, and is able to distinguish between highly irregular or uncorrelated 
complex physical signals. MSE have advantage to measure the irregularity time series under different time scales ${ }^{51}$. It's designed to reflect the degree of health condition of a biological system by its output physiological signals ${ }^{53}$, which is particularly developed to analyze nonlinear and non-stationary constant signals, and their relationship. It has been hypothesized that as part of complexity physiologic systems, EEG and HRV have similar changing trend on the same time scale $^{41}$. However, experimental evidence to support this hypothesis is limited. In this study, we are going to evaluate whether the widely utilized complexity-based metrics- MSE is sensitive and informative metric for characterizing the impact of qigong on complexity in cardiovascular and nervous systems ${ }^{54-56}$.

Aforementioned we briefly summarized our studies protocol that support the value of complexity-based biomarkers in characterizing correlation in cardiovascular and nervous dynamics.This study will prove its mind body effects by to test the methods and calculate a sample size for future randomized trials to investigate further the clinical value of qigong in mind body diseases.

\section{List of abbreviations}

Abbreviations

CMQ

TCPSQ

EEG

HRV

ECG

MSE

BUCM

CRC

LF

ICA

HR

ANOVA

\section{Chinese Medical Qigong}

Three-circle Post Standing qigong

Electroencephalogram

Heart rate variability

Electrocardiogram

Multiscale Entropy

Beijing University of Chinese Medicine

clinical research coordinator

low frequency in HRV

Independent Component Analysis

heart rate

one-way analysis of variance

\section{Declarations}

\section{Ethics and dissemination}

The protocol has been approved by the institutional review boards of Beijing University of Chinese Medicine, where the study will take place(approval number: 2018BZHYLL0109).

\section{Trail registration:}


This study has been registered with the "Chinese Clinical Trail Registry" , Republic of China, which is a registry in the WHO Registry Network. Register number: ChiCTR-Bon-17010840.

\section{Consent for publication}

Not applicable

\section{Availability of data and materials}

Data sharing is not applicable to this article as no datasets were generated or analysed during the current study.

\section{Competing interests}

The authors declare that they have no competing interests.

\section{Funding}

This study was supported by the National Nature Science Foundation of China.(No.81674043).

\section{Author contributions}

Conceptualization: Jiaxuan Lyu, Yulong Wei.

Data curation: Hangyu Li, Jingjing Dong, Xinzheng Zhang.

Investigation: Jiaxuan Lyu, Tianyang Tan.

Methodology: Jiaxuan Lyu, Yulong Wei.

Project administration: Jiaxuan Lyu, Yulong Wei.

Supervision: Jiaxuan Lyu, Yulong Wei.

Validation: Jiaxuan Lyu, Qingchuan Hu.

Visualization: Jiaxuan Lyu, Yulong Wei, Tianyang Tan, Qingchuan Hu, Hangyu Li, Jinging Dong, Xinzheng Zhang.

Writing - original draft: Jiaxuan Lyu

Writing - review \& editing: Yulong Wei.

Jiaxuan Lyu orcid: 0000-0002-5410-6957

\section{Acknowledgments}

Not applicable

\section{References}

1.Oberoi D, Piedalue KL, Pirbhai H, et al. Factors related to dropout in integrative oncology clinical trials: interim analysis of an ongoing comparative effectiveness trial of mindfulness-based cancer recovery and Tai chi/Qigong for cancer health (The MATCH study). BMC Res Notes. 2020 Jul 17;13(1):342. 
2.Garland S N , Mahon K, Irwin M R . Integrative Approaches for Sleep Health in Cancer Survivors[J]. The Cancer Journal, 2019, 25(5):337-342.

3.Carlson LE, Oberoi DV, Qureshi M, Subnis U. Integrative Oncology Trials in the Real World: Assessing the Pragmatism of an Ongoing Integrative Oncology Trial of Mindfulness and T'ai Chi/Qigong. J Altern Complement Med. 2018 Sep/Oct;24(9-10):926-932.

4.Liu P, You J, Loo WTY, Sun Y, He Y, Sit H, Jia L, Wong M, Xia Z, Zheng X, Wang Z, Wang N, Lao L, Chen J. The efficacy of Guolin-Qigong on the body-mind health of Chinese women with breast cancer: a randomized controlled trial. Qual Life Res. 2017 Sep;26(9):2321-2331.

5.Larkey L, Kim W, James D, Kishida M, Vizcaino M, Huberty J, Krishnamurthi N. Mind-Body and Psychosocial Interventions May Similarly Affect Heart Rate Variability Patterns in Cancer Recovery: Implications for a Mechanism of Symptom Improvement. Integr Cancer Ther. 2020 Jan-Dec;19:1534735420949677.

6.Chen $\mathrm{CH}$, Hung KS, Chung YC, Yeh ML. Mind-body interactive qigong improves physical and mental aspects of quality of life in inpatients with stroke: A randomized control study. Eur J Cardiovasc Nurs. 2019 Dec;18(8):658-666.

7.Walter AA, Van Puymbroeck M, Bosch P, Schmid AA. Complementary and integrative health interventions in post-stroke rehabilitation: a systematic PRISMA review. Disabil Rehabil. 2020 Oct 12:1-10.

8.Kim H, Kim YL, Lee SM. Effects of therapeutic Tai Chi on balance, gait, and quality of life in chronic stroke patients. Int J Rehabil Res. 2015 Jun;38(2):156-61.

9.Luo XC, Zhou J, Zhang YG, Liu YY, Li JJ, Zheng Z, Tong F, Feng F. Effects of Tai Chi Yunshou on upper limb function and balance in stroke survivors: A protocol for systematic review and meta analysis. Medicine (Baltimore). 2020 Jul 17;99(29):e21040.

10.Chan WN, Tsang WW. The effect of Tai Chi training on the dual-tasking performance of stroke survivors: a randomized controlled trial. Clin Rehabil. 2018 Aug;32(8):1076-1085.

11.Li L, Cheng S, Wang G, Duan G, Zhang Y. Tai chi chuan exercises improve functional outcomes and quality of life in patients with primary total knee arthroplasty due to knee osteoarthritis. Complement Ther Clin Pract. 2019 May;35:121-125.

12.Osypiuk K, Ligibel J, Giobbie-Hurder A, Vergara-Diaz G, Bonato P, Quinn R, Ng W, Wayne PM. Qigong Mind-Body Exercise as a Biopsychosocial Therapy for Persistent Post-Surgical Pain in Breast Cancer: A Pilot Study. Integr Cancer Ther. 2020 Jan-Dec;19:1534735419893766.

13.Park JE, Kim JE, Jung S, Kim A, Park H, Hong S. The Effect of Dongeui Qigong for Prehypertension and Mild Essential Hypertension. Evid Based Complement Alternat Med. 2017;2017:4274538.

14.Cheng T. O. Tai Chi: the Chinese ancient wisdom of an ideal exercise for cardiac patients. International Journal of Cardiology. 2007;117(3):293-295.

15. Blake H., Hawley H. Effects of Tai Chi exercise on physical and psychological health of older people. Current Aging Science. 2012;5(1):19-27. 
16.Lan C., Chen S. Y., Lai J. S., Wong A. M. Tai Chi Chuan in medicine and health promotion. Evidence-Based Complementary and Alternative Medicine.

17. Huston P., McFarlane B. Health benefits of Tai Chi: what is the evidence? Canadian Family Physician. 2016;62:881-890.

18.Yu A. P., Tam B. T., Lai C. W., et al. Revealing the neural mechanisms underlying the beneficial effects of Tai Chi: a Neuroimaging perspective. The American Journal of Chinese Medicine.

19. Lan C., Chen S. Y., Wong M. K., Lai J. S. Tai Chi Chuan exercise for patients with cardiovascular disease. Evidence-Based Complementary and Alternative Medicine. 2013;2013:9.

20.Wang J., Feng B., Yang X. C., et al. Tai Chi for essential hypertension. Evidence-Based Complementary and Alternative Medicine. 2013;2013:10.

21.Chen D. Effect of health Qigong Mawangdui Daoyinshu on blood pressure of individuals with essential hypertension. Journal of the American Geriatrics Society. 2016;64(7):1513-1515.

22.Jin H. L., Pang J. J. Effects of Tai Chi on 24-hour ambulatory blood pressure and vascular endothelial function in patients with primary hypertension of stage I. Chinese Journal of Sports Medicine. 2016;35(3):20-23.

23.Xie H. J., Bai C. Q. Gaseous signal molecular mechanism of Tai Chi intervention in aged essential hypertension. Journal of Wuhan Institute of Physical Education. 2014;48(2):51-54.

24.Huang C.-L., Tai Y.-K., Yang Y.-H., Wang R.-H. Efficacy of five-element gymnastics in glucose and lipid control in taiwanese patients with type 2 diabetes. Research in Nursing \& Health. 2012;35(4):419-429.

25.Freire M. D. M., Alves C. Therapeutic Chinese exercises (Qigong) in the treatment of type 2 diabetes mellitus: A systematic review. Diabetes \& Metabolic Syndrome: Clinical Research \& Reviews. 2013;7(1):56-59.

26.Yang J., Jing-ying L., Wenliang L., Lina M., Hong Z. Meta-analysis on effects of Baduanjin on patients with type 2 diabetes mellitus. China Journal of Traditional Chinese Medicine and Pharmacy. 2015:1307-1309.

27.Yu T.-T., Yu X.-L., Zeng L.-M., Zhou X., Zhao R.-H. Baduanjin for diabetes: A systematic review. Chinese Journal of Evidence-Based Medicine. 2014;14(3):341-348.

28.Fan M , Yang A C , Fuh J L, et al. Topological Pattern Recognition of Severe Alzheimer's Disease via Regularized Supervised Learning of EEG Complexity[J]. Frontiers in Neuroscience, 2018, 12 .

29. Costa M , Goldberger A L , Peng C K . Multiscale Entropy Analysis of Complex Physiologic Time Series[J]. Physical Review Letters, 2002.

30.Costa M D , Peng C K , Goldberger A L . Multiscale Analysis of Heart Rate Dynamics: Entropy and Time Irreversibility Measures[J]. Cardiovascular Engineering, 2008, 8(2):88-93.

31.Ma Y, Wu C W, Peng C K, et al. Complexity-Based Measures of Heart Rate Dynamics in 
Older Adults Following Long- and Short-Term Tai Chi Training: Cross-sectional and Randomized Trial Studies[J]. entific Reports, 2019, 9(1):7500.

32.Ma Y , Mccraty R . Heart Rate Variability in Mind-Body Interventions[J]. Complementary Therapies in Medicine, 2016:S0965229916300565.

33.Wu S D , Wu C W, Lee K Y , et al. Modified multiscale entropy for short-term time series analysis[J]. Physica A Statal Mechanics \& Its Applications, 2013, 392(23):5865-5873.

34.Wu S D , Wu C W, Lin S G, et al. Analysis of complex time series using refined composite multiscale entropy[J]. Physics Letters A, 2014, 378(20):1369-1374.

35.A Y Y , B P S , F A C A D E , et al. Multiscale joint permutation entropy for complex time series[J]. Physica A: Statal Mechanics and its Applications, 2019, 515:388-402.

36.Liang W K , Lo M T , Yang A C , et al. Revealing the brain's adaptability and the transcranial direct current stimulation facilitating effect in inhibitory control by multiscale entropy[J]. Neuroimage, 2014, 90:218-234.

37.Costa M , Goldberger A L , Peng C K . Multiscale entropy to distinguish physiologic and synthetic RR time series.[J]. Comput Cardiol, 2002, 29(29):137-140.

38.Mao X , Shang P, Yang A C , et al. Multiscale cumulative residual distribution entropy and its applications on heart rate time series[J]. Nonlinear Dynamics, 2020, 101(4):1-12.

39.Costa M D , Peng C K , Goldberger A L . Multiscale Analysis of Heart Rate Dynamics: Entropy and Time Irreversibility Measures[J]. Cardiovascular Engineering, 2008, 8(2):88-93.

40.Fan M, Yang A C , Fuh J L, et al. Topological Pattern Recognition of Severe Alzheimer's Disease via Regularized Supervised Learning of EEG Complexity[J]. Frontiers in Neuroscience, 2018, 12.

41.Yeh J R , Peng C K , Lo M T , et al. Investigating the interaction between heart rate variability and sleep EEG using nonlinear algorithms[J]. Journal of Neuroence Methods, 2013, 219(2):233-239.

42.Rodriguez E , Echeverria J C , Alvarez-Ramirez J . Detrended fluctuation analysis of heart intrabeat dynamics[J]. Physica A Statal Mechanics \& Its Applications, 2007, 384(2):429-438.

43. Willson K , Francis D P, Wensel R, et al. Relationship between detrended fluctuation analysis and spectral analysis of heart-rate variability[J]. Physiological Measurement, 2002, 23(2):385-401.

44.Guo Y, Xu M, Zhang J , et al. The effect of Three-Circle Post Standing (Zhanzhuang) Qigong on the physical and psychological well-being of college students[J]. other, 2018, 97(38).

45. Lyu Jiaxuan. Analysis of the effect on Three-circle Post Standing Qigong's function of psychosomatic balance on college students' suffer from spinal imbalance. (Master's degree), Beijing University of Chinese Medicine.2018.

46. Yan Jian. Study on physiological mechanisms to improve the anxiety status of college students by using heart rate variability analysis. (Master's degree), Beijing University of Traditional Chinese Medicine, 2019. 
47.Machin, D., Campbell, M., Fayers, P., and Pinol, A. Sample Size Tables for Clinical Studies,Edition. Blackwell Science. Malden, MA.1997.

48.Zar, Jerrold H. Biostatistical Analysis (Second Edition). Prentice-Hall. Englewood Cliffs, New Jersey.1984.

49.Liu TJ, Zhang WC. Traditional Chinese medicine qigong. China Press Tradit Chin Med 2016;114-7.

50.Wei YL. Chinese medicine qigong training guidance. China Press Tradit Chin Med 2016;120-35.

51.Wayne P M , Gagnon M M , Macklin E A, et al. The Mind Body-Wellness in Supportive Housing (Mi-WiSH) study: Design and rationale of a cluster randomized controlled trial of Tai Chi in senior housing[J]. Contemporary Clinical Trials, 2017, 60:96-104.

52.A P M W , C B M B , C V N B , et al. A systems biology approach to studying Tai Chi, physiological complexity and healthy aging: Design and rationale of a pragmatic randomized controlled trial[J]. Contemporary Clinical Trials, 2013, 34( 1):21-34.

53.Hsu C F , Chao H H, Yang A C , et al. Discrimination of Severity of Alzheimer's Disease with Multiscale Entropy Analysis of EEG Dynamics[J]. Applied ences, 2020, 10(4):1244.

54.Yang A C, Wang S J , Lai K L, et al. Cognitive and neuropsychiatric correlates of EEG dynamic complexity in patients with Alzheimer's disease.[J]. Progress in Neuropsychopharmacology \& Biological Psychiatry, 2013, 47:52-61.

55.Hsu C F , Chao H H, Yang A C , et al. Discrimination of Severity of Alzheimer's Disease with Multiscale Entropy Analysis of EEG Dynamics[J]. Applied ences, 2020, 10(4):1244.

56.Jiangkuan C , Cong L , Chung-Kang P , et al. Topological reorganization of EEG functional network is associated with the severity and cognitive impairment in Alzheimer's disease[J]. PHYSICA A-STATISTICAL MECHANICS AND ITS APPLICATIONS, 2018:S0378437118311609-. 

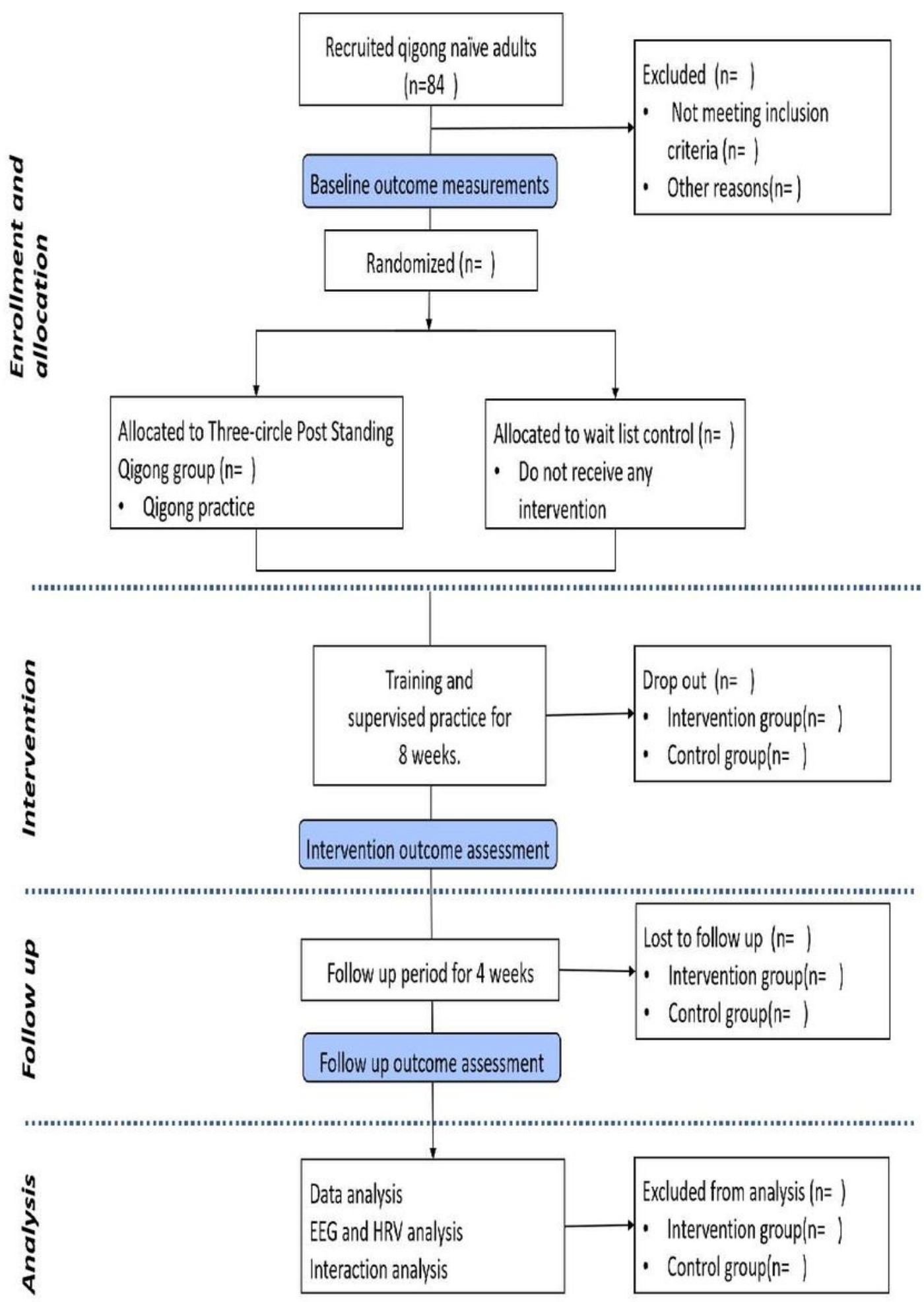

\section{Figure 1}

Flowchart of the study design 